TOKYo J. Math.

VoL. 7 , No. 1,1984

\title{
Non-Expansive Attractors with Specification
}

\author{
Motomasa KOMURO \\ Tokyo Metropolitan University \\ (Communicated by K. Ogiue)
}

\section{Introduction}

Let $f: Y \rightarrow Y$ be a continuous surjection of a compact metric space $Y$. The inverse limit of $f$ induces a compact metric space $\bar{Y}$ and a homeomorphism $\bar{f}$ of $\bar{Y} . \quad(\bar{Y}, \bar{f})$ is called the natural extension of $f$. As R. Williams proved in [12], if a 1-dimensional branched manifold $Y$ admits an expanding immersion $g: Y \rightarrow Y$, then $Y$ has no endpoints. Moreover $(\bar{Y}, \bar{g})$ is topologically conjugate to an attractor of some Axiom $A$ diffeomorphism. But some attractors, as Hénon's attractors, resemble the natural extension $(\bar{I}, \bar{f})$ of a continuous surjection $f$ of an interval $I$ with endpoints. It is a problem whether there exist any diffeomorphisms which have an attractor topologically conjugate to $(\bar{I}, \bar{f})$.

For the continuous surjection $f(x)=1-|2 x-1|$ on the interval $I=[0,1]$, we show in this paper that there exists a diffeomorphism of the 3-sphere which has an attractor topologically conjugate to $(\bar{I}, \bar{f})$. Furthermore we show that $(\bar{I}, \bar{f})$ satisfies not expansiveness but specification (these properties have been used in papers [1,2], [3, 4], [5], [8] and [10] on ergodic theory). To realize the attractor in the 3 -sphere, our key ingregient is in constructing a fine foliation of a closed 3-ball.

\section{§1. Definitions and results.}

Let $X=(X, d)$ be a compact metric space and $\sigma$ a homeomorphism of $X$ (i.e. from $X$ onto itself). By $R, Z$ and $N$ we denote the set of real numbers, the set of integers and the set of positive integers respectively. $(X, \sigma)$ is expansive if there exists a $\delta>0$ such that, for every pair of distinct points $x, y \in X$, there is an $n \in Z$ with $d\left(\sigma^{n} x, \sigma^{n} y\right)>\delta . \quad(X, \sigma)$ is said to satisfy specification if the following holds; for every $\varepsilon>0$ there exists an integer $K=K(\varepsilon)>0$ such that, for every $k \geqq 1$, for every 
$k$ points $x_{1}, \cdots, x_{k} \in X$, for every integers

$$
a_{1} \leqq b_{1}<a_{2} \leqq b_{2}<\cdots<a_{k} \leqq b_{k}
$$

with

$$
a_{i+1}-b_{i} \geqq K \quad(1 \leqq i \leqq k-1)
$$

and for every integer $p$ with $p \geqq b_{k}-a_{1}+K$, there exists a point $x \in X$ with $\sigma^{p} x=x$ such that

$$
d\left(\sigma^{n} x, \sigma^{n} x_{i}\right)<\varepsilon \quad \text { for } \quad a_{i} \leqq n \leqq b_{i}, 1 \leqq i \leqq k .
$$

$(X, \sigma)$ is said to be topologically transitive if $\left\{\sigma^{n} x: n \in Z\right\}$ is dense in $X$ for some $x \in X$. If $(X, \sigma)$ satisfies specification, then it is clearly topologically transitive. Let $\sigma_{1}$ be a homeomorphism of a compact metric space $X_{1} . \quad(X, \sigma)$ and $\left(X_{1}, \sigma_{1}\right)$ are said to be topologically conjugate to each other if there exists a homeomorphism $\varphi$ from $X$ onto $X_{1}$ such that $\varphi \circ \sigma=\sigma_{1} \circ \varphi$. The topological conjugacy is an equivalent relation under which specification, topological transitivity and expansiveness are preserved.

Let $Y=(Y, d)$ be a compact metric space and $f: Y \rightarrow Y$ a continuous surjection. $(Y, f)$ is said to satisfy positive specification if it satisfies the condition of specification for $a_{1} \geqq 0$. We define the metric $\bar{d}$ of the direct product space $Y^{N}$ by $\bar{d}(\bar{x}, \bar{y})=\sum_{i=1}^{\infty} 2^{-i} d\left(x_{i}, y_{i}\right)$ for $\bar{x}=\left(x_{i}\right)_{1}^{\infty}$ and $\bar{y}=$ $\left(y_{i}\right)_{1}^{\infty}$ in $Y^{N}$. The compact subset $X$ of $Y^{N}$ is defined by

$$
X=\left\{\bar{x} \in Y^{N}: f\left(x_{i+1}\right)=x_{i}, i \in N\right\} .
$$

Let $\sigma: X \rightarrow X$ be the homeomorphism defined by $\sigma(\bar{x})=\left(f x_{1}, f x_{2}, f x_{3}, \cdots\right)=$ $\left(f x_{1}, x_{1}, x_{2}, \cdots\right)$ for $\bar{x}=\left(x_{1}, x_{2}, \cdots\right) \in X . \quad(X, \sigma)$ is called the natural extension of $(Y, f)$, and it is denoted by $(X, \sigma)=\lim (Y, f)$.

Let $g$ be a diffeomorphism of a compact manifold $M$. A $g$-invariant subset $\Lambda$ of $M$ is said an attractor of $g$ if there exists a closed neighborhood $W$ of $\Lambda$ such that

(i) $g(W) \subset \operatorname{int}(W)$,

(ii) $\Lambda=\bigcap_{n \geq 0} g^{n}(W)$ and

(iii) $g \mid \Lambda: \Lambda \rightarrow \Lambda$ is topologically transitive.

We denote by $(\Lambda, g)$ the restriction of $(M, g)$ to an attractor $\Lambda$. Our main results are stated in the theorems below:

THEOREM 1. Let $I=[1,0]$ be a compact interval with the euclidian metric, and $f: I \rightarrow I$ a continuous surjection defined by $f(x)=1-|2 x-1|$ $(x \in I)$. Let $(X, \sigma)$ be the natural extension of $(I, f)$. Then the following 
holds:

and
(A) $(X, \sigma)$ is not expansive,
(B) $(X, \sigma)$ satisfies specification

(C) each point of $X$ has a neighborhood which is homeomorphic to the product of a compact interval and a Cantor set.

THEOREM 2. Let $(X, \sigma)$ be as in Theorem 1. Then there exists a $C^{1}$ diffeomorphism $g$ of the 3-sphere $S^{s}$ which has an attractor $\Lambda$ such that $(\Lambda, g)$ is topologically conjugate to $(X, \sigma)$.

\section{§2. Proof of Theorem 1.} $x, y \in I$.

We denote by $d$ the euclidian metric of $I$; i.e. $d(x, y)=|x-y|$ for
$\in I$.

(I) Proof of (A). Let $1 / 2>\varepsilon>0$ be given. For each $i \geqq 1$, we put $x_{i}=2^{-i}(1-\varepsilon)$ and $y_{i}=2^{-i}(1+\varepsilon)$. Then $\bar{x}=\left(x_{1}, x_{2}, \cdots\right)$ and $\bar{y}=\left(y_{1}, y_{2}, \cdots\right)$ are distinct points of $X$, because $x_{1} \neq y_{1}, f\left(x_{i+1}\right)=x_{i}$ and $f\left(y_{i+1}\right)=y_{i}$ for each $i \geqq 1$. To prove (A), it is enough to show that $d\left(\sigma^{n} \bar{x}, \sigma^{n} \bar{y}\right) \leqq \varepsilon$ for every $n \in Z$. Let $n \in Z$ be given. If $n \geqq 0$, using the fact that $f^{i}\left(x_{1}\right)=f^{i}\left(y_{1}\right)$ for every $i \geqq 1$, we have

$$
\begin{aligned}
\bar{d}\left(\sigma^{n} \bar{x}, \sigma^{n} \bar{y}\right) & =\bar{d}\left(\left(f^{n} x_{1}, f^{n-1} x_{1}, \cdots, f x_{1}, x_{1}, x_{2}, \cdots\right),\right. \\
& \left.\quad\left(f^{n} y_{1}, f^{n-1} y_{1}, \cdots, f y_{1}, y_{1}, y_{2}, \cdots\right)\right) \\
& =\sum_{i=1}^{\infty} 2^{-(n+i)} d\left(x_{i}, y_{i}\right) \\
& =2^{-n+1} \sum_{i=1}^{\infty} 2^{-2 i} \\
& =2^{-n+1} \varepsilon / 3<\varepsilon .
\end{aligned}
$$

If $n<0$, we have

$$
\begin{aligned}
\bar{d}\left(\sigma^{n} \bar{x}, \sigma^{n} \bar{y}\right) & =\bar{d}\left(\left(x_{1-n}, x_{2-n}, \cdots\right),\left(y_{1-n}, y_{2-n}, \cdots\right)\right) \\
& =\sum_{i=1}^{\infty} 2^{-i} d\left(x_{i-n}, y_{i-n}\right) \\
& =2^{n+1} \varepsilon / 3<\varepsilon .
\end{aligned}
$$

Therefore $(X, \sigma)$ is not expansive.

(II) Proof of (B). To prove (B), it is enough to prove the next two propositions.

Proposition 2.1. If $(I, f)$ satisfies positive specification, then 
$(X, \sigma)=\lim _{\leftarrow}(I, f)$ satisfies specification.

Proposition 2.2. $(I, f)$ satisfies positive specification.

Proof of Proposition 2.1. Assume that $(I, f)$ satisfies positive specification. Let $\varepsilon>0$ be given. Choose a positive integer $N$ such that $2^{-N}<\varepsilon / 2$. Let $K^{\prime}=K^{\prime}(\varepsilon / 2)>0$ be as in the definition of positive specification. Put $K=K^{\prime}+N$ and take any integer $k \geqq 1$. Let $\bar{x}_{1}, \bar{x}_{2}, \cdots, \bar{x}_{k} \in X$ be given, as well as integers $a_{1} \leqq b_{1}<a_{2} \leqq b_{2}<\cdots<a_{k} \leqq b_{k}$ and $p$ with $a_{i+1}-b_{i} \geqq K$ $(1 \leqq i \leqq k-1)$ and $p \geqq b_{k}-a_{1}+K$. We have to show that there exists a $\bar{y} \in X$ with $\sigma^{p} \bar{y}=\bar{y}$ such that $\bar{d}\left(\sigma^{n} \bar{y}, \sigma^{n} \bar{x}_{i}\right)<\varepsilon$ for every $a_{i} \leqq n \leqq b_{i}$ and $1 \leqq$ $i \leqq k$. To do this we consider two cases separately.

Case (i): $a_{1} \geqq 0$. For each $1 \leqq i \leqq k$, the point $\bar{x}_{i}$ is expressed by $\bar{x}_{i}=$ $\left(x_{1}^{i}, x_{2}^{i}, \cdots\right)$ where $x_{j}^{i} \in I(j \in N)$. Note that $a_{i+1}-\left(b_{i}+N\right) \geqq K^{\prime}(1 \leqq i \leqq k-1)$ and $p \geqq\left(b_{k}+N\right)-a_{1}+K^{\prime}$. Since $(I, f)$ satisfies positive specification, for $x_{N}^{i} \in I(1 \leqq i \leqq k)$, for $a_{1} \leqq b_{1}+N<a_{2} \leqq b_{2}+N<\cdots<a_{k} \leqq b_{k}+N$ and for $p$, there exists $y \in I$ with $f^{p} y=y$ such that $d\left(f^{n} y, f^{n} x_{N}^{i}\right)<\varepsilon / 2$ for every $a_{i} \leqq n \leqq b_{i}+N$ and $1 \leqq i \leqq k$. Define $\bar{y} \in X$ by

$$
\bar{y}=\left(f^{N-1} y, f^{N-2} y, \cdots, f y, y, f^{p-1} y, f^{p-2} y, \cdots, f y, y, f^{p-1} y, \cdots\right) \text {. }
$$

Then $\bar{y}$ satisfies $\sigma^{p} \bar{y}=\bar{y}$. For each $1 \leqq i \leqq k$, since $x_{j}^{i}=f\left(x_{j+1}^{i}\right)$ for every $j \in N, \bar{x}_{i}$ is expressed by

$$
\bar{x}_{i}=\left(f^{N-1} x_{N}^{i}, \cdots, f x_{N}^{i}, x_{N}^{i}, x_{N+1}^{i}, \cdots\right) .
$$

Since $\operatorname{diam}(I)=1$, we have, for every $a_{i} \leqq n \leqq b_{i}$,

$$
\begin{aligned}
& \bar{d}\left(\sigma^{n} \bar{y}, \sigma^{n} \bar{x}_{i}\right)= \bar{d}\left(\left(f^{n+N-1} y, \cdots, f^{n+1} y, f^{n} y, f^{n+p-1} y, \cdots\right),\right. \\
&\left.\left(f^{n+N-1} x_{N}^{i}, \cdots, f^{n+1} x_{N}^{i}, f^{n} x_{N}^{i}, f^{n} x_{N+1}^{i}, \cdots\right)\right) \\
& \leqq \sum_{j=1}^{N} 2^{-j} d\left(f^{n+N-j} y, f^{n+N-j} x_{N}^{i}\right)+\sum_{j=N+1}^{\infty} 2^{-j} \\
&<\varepsilon / 2+1 / 2^{N}<\varepsilon .
\end{aligned}
$$

Case (ii): $a_{1}<0$. Put $\bar{x}_{i}^{\prime}=\sigma^{a_{1}} \bar{x}_{i}, a_{i}^{\prime}=a_{i}-a_{1}$ and $b_{i}^{\prime}=b_{i}-a_{1}(1 \leqq i \leqq k)$. Note that $a_{i+1}^{\prime}-\left(b_{i}^{\prime}+N\right) \geqq K^{\prime}$ and $p \geqq\left(b_{k}^{\prime}+N\right)-a_{1}^{\prime}+K^{\prime}$. Apply the case (i) to $\bar{x}_{i}^{\prime} \in X(1 \leqq i \leqq k), 0=a_{1}^{\prime} \leqq b_{1}^{\prime}+N<a_{2}^{\prime} \leqq b_{2}^{\prime}+N<\cdots<a_{k}^{\prime} \leqq b_{k}^{\prime}+N$ and $p$. Then we get $\bar{y}^{\prime} \in X$ with $\sigma^{p} \bar{y}^{\prime}=\bar{y}^{\prime}$ such that $\bar{d}\left(\sigma^{n} \bar{y}^{\prime}, \sigma^{n} \bar{x}_{i}^{\prime}\right)<\varepsilon$ for $a_{i}^{\prime} \leqq n \leqq b_{i}^{\prime}, 1 \leqq$ $i \leqq k$. Put $\bar{y}=\sigma^{-a_{1}} \bar{y}^{\prime}$, then this is a required point. Proposition 2.1 is proved.

To prove Proposition 2.2, we prepare two lemmas.

LEMMA 2.3. Let $Y$ be a compact interval and $\xi: Y \rightarrow R$ a continuous 
map. Let a closed interval $J \subset \xi(Y)$ be given. Then there exists a closed interval $J^{\prime} \subset Y$ such that $\xi\left(J^{\prime}\right)=J$.

Proof. Put $J=[a, b]$. If $a=b$, the assertion is trivial. Suppose $a<b$. Then there are $c, d \in Y$ such that $\xi(c)=a$ and $\xi(d)=b$. If $c<d$, put $q=\inf \{x \in[c, d]: \xi(x)=b\}$ and $p=\sup \{x \in[c, q]: \xi(x)=a\}$. Otherwise, put $p=\sup \{x \in[d, c]: \xi(x)=b\}$ and $q=\inf \{x \in[p, c]: \xi(x)=a\} . \quad$ In any case, by the intermediate-value theorem, we have $\xi([p, q])=J$.

For $x \in I$ and $\varepsilon>0$, define $I(x, \varepsilon)=\{y \in I: d(x, y) \leqq \varepsilon\}$.

LEMMA 2.4. Let $\varepsilon>0$ be given.

(i) For every $x \in I$ and $n \geqq 0$, it follows that

$$
f^{n}\left(I\left(x, \varepsilon / 2^{n}\right)\right)=I\left(f^{n} x, \varepsilon\right)
$$

and

$$
d\left(f^{i} x, f^{i} y\right) \leqq \varepsilon \quad \text { for every } \quad 0 \leqq i \leqq n \quad \text { and } y \in I\left(x, \varepsilon / 2^{n}\right) .
$$

(ii) There exists an integer $K=K(\varepsilon)>0$ such that the following holds: for every $x \in I$, for every closed interval $I^{\prime} \subset I$ and for every $n \geqq K$, there is a closed interval $J \subset I(x, \varepsilon)$ such that $f^{n}(J)=I^{\prime}$.

Proof. By the definition of $f$, one has $f(I(x, \varepsilon))=I(f x, 2 \varepsilon)$ for every $x \in I$ and every $\varepsilon>0$ (not necessary $\varepsilon<1$ ). Applying this to $I\left(x, \varepsilon / 2^{n}\right.$ ) repeatedly, we get (i). To see (ii), choose $K>0$ such that $2^{-K}<\varepsilon$. Then, since $2^{K} \varepsilon \geqq 1$, it follows that $f^{n}(I(x, \varepsilon))=I\left(f^{n} x, 2^{n} \varepsilon\right)=I$ for every $x \in I$ and every $n \geqq K$. Replacing $\xi$ in Lemma 2.3 by $f^{n}$, we get (ii).

Proof of Proposition 2.2. Let $\varepsilon>0$ be given. Choose a number $\varepsilon^{\prime}$ with $0<\varepsilon^{\prime}<\varepsilon$. Let $k=k\left(\varepsilon^{\prime}\right)>0$ be an integer as in Lemma 2.4 (ii). Take any $k \geqq 1$. Let $x_{1}, \cdots, x_{k} \in X$ be given, as well as integers $0 \leqq a_{1} \leqq$ $b_{1}<a_{2} \leqq b_{2}<\cdots<a_{k} \leqq b_{k}$ and $p$ with $a_{i+1}-b_{i} \geqq K(1 \leqq i \leqq k-1)$ and $p \geqq b_{k}-$ $a_{1}+K$. Put $a_{k+1}=p+a_{1}$.

In order to find an interval $I_{1} \subset I \subset\left(f^{a_{1}} x_{1}, \varepsilon^{\prime}\right)$ such that $f^{p}\left(I_{1}\right) \supset I_{1}$, put $I_{k+1}=I\left(f^{a_{1}} x_{1}, \varepsilon^{\prime}\right)$. Then $I_{i}(i \leqq k)$ is determined recursively as follows. By Lemma 2.4 (ii), there is an interval $J_{i} \subset I\left(f^{b_{i}} x_{i}, \varepsilon^{\prime}\right)$ such that $f^{a_{i+1}-b_{i}}\left(J_{i}\right)=$ $I_{i+1}$. Since $f^{b_{i}-a_{i}}\left(I\left(f^{a_{i}} x_{i}, \varepsilon^{\prime} / 2^{b_{i}-a_{i}}\right)\right)=I\left(f^{b_{i}} x_{i}, \varepsilon^{\prime}\right)$ (by Lemma 2.4 (i)), there exists an interval $I_{i} \subset I\left(f^{a_{i}} x_{i}, \varepsilon^{\prime} / 2^{b_{i}-a_{i}}\right)$ such that $f^{b_{i}-a_{i}}\left(I_{i}\right)=J_{i}$ (by Lemma 2.3).

Since $f^{a_{i+1}-a_{i}}\left(I_{i}\right)=I_{i+1}$ for $1 \leqq i \leqq k$, one has $I_{k+1}=f^{a_{k+1}-a_{1}}\left(I_{1}\right)=f^{p}\left(I_{1}\right)$. Note that $I_{1} \subset I\left(f^{a_{1}} x_{1}, \varepsilon^{\prime}\right)=I_{k+1}$. By the intermediate-value theorem, theer exists a $y \in I_{1}$ such that $f^{p} y=y$. Put $x=f^{p-a_{1}} y$. Clearly $f^{p} x=x$ holds. 
For every $1 \leqq i \leqq k$ and $a_{i} \leqq n \leqq b_{i}$, one has $f^{n} x=f^{n-a_{1}} y \in f^{n-a_{1}}\left(I_{1}\right)=$ $f^{n-a_{i}}\left(I_{i}\right) \subset f^{n-a_{i}} I\left(f^{a_{i}} x_{i}, \varepsilon^{\prime} / 2^{b_{i}-a_{i}}\right) \subset I\left(f^{n} x_{i}, \varepsilon^{\prime}\right)$; i.e., $d\left(f^{n} x, f^{n} x_{i}\right) \leqq \varepsilon^{\prime}<\varepsilon$. This means that $(I, f)$ satisfies positive specification. The proof is completed.

(III) Proof of (C). Let $\bar{a}=\left(a_{1}, a_{2}, \cdots\right) \in X$ be given. Denote by $J_{1}$ the 1/4-closed neighborhood of $a_{1}$ in $I$. If $J \subset I$ is an interval such that $\operatorname{diam}(J) \leqq 1 / 2$, then $f^{-2}(J)$ has at least two connected components and the diameter of each connected component of $f^{-2}(J)$ is not greater than $(1 / 2) \operatorname{diam}(J)$. Hence, for $J_{n}=f^{-2(n-1)}\left(J_{1}\right) \quad(n \geqq 1)$, there exists a homeomorphism $\psi_{n}: J_{n} \rightarrow I \times F_{n}$ where $F_{n}$ is a finite set with card $\left(F_{n}\right) \geqq 2^{n-1}$. Put $V_{0}=\left\{\bar{x} \in X: x_{1} \in J_{1}\right\}$. Clearly $V_{0}$ is a neighborhood of $\bar{a}$, and this is expressed by the inverse limit of the sequence

$$
J_{1} \longleftarrow f^{2} J_{2} \longleftarrow f^{2} J_{3} \stackrel{f^{2}}{\cdots} .
$$

Therefore $V_{0}$ is homeomorphic to the inverse limit of the sequence

$$
I \times F_{1} \overleftarrow{\Psi_{1}} I \times F_{2} \overleftarrow{\Psi_{2}} I \times F_{3} \overleftarrow{\Psi_{3}} \cdots,
$$

where $\psi_{n}=\psi_{n} \circ f^{2} \circ \psi_{n+1}^{-1}(n \geqq 1)$. This implies that $V_{0}$ is homeomorphic to the product of $I$ and a Cantor set. The proof is completed.

\section{§3. Proof of Theorem 2.}

Let $f(x)=1-|2 x-1|$ as before. Define the continuous map $h:(-1 / 2$, $3 / 2) \rightarrow R$ by

$$
h(x)= \begin{cases}f(x)-(2 \pi)^{-1} \sin (2 \pi x) & (-1 / 2<x \leqq 1 / 2) \\ f(x)+(2 \pi)^{-1} \sin (2 \pi x) & (1 / 2<x<3 / 2)\end{cases}
$$

Clearly $h$ satisfies the following.

(L.1) (i) $h(0)=0, h(1 / 2)=1$ and $h(x)=h(1-x)$ for $-1 / 2<x<3 / 2$.

(ii) $h(-x)=-h(x)$ for $-1 / 2<x<1 / 2$.

(iii) $h^{\prime}(0)=1$ and $h^{\prime}(x)>1$ for $x \in(-1 / 2,1 / 2)-\{0\}$.

(iv) $h(x)=x+o\left(x^{2}\right)$.

Here $h^{\prime}$ denotes the derivative of $h$, and $o(t)$ means a function such that $o(t) / t \rightarrow 0$ as $t \rightarrow 0$.

Note that the restriction of $h$ to $I$ is a continuous map from $I$ onto itself. Let $\left(X_{h}, \sigma_{h}\right)=\lim (I, h)$ and $(X, \sigma)=\lim (I, f)$. Clearly Theorem 2 is obtained from the $\overleftarrow{\text { next }}$ two propositions.

Proposition 3.1. $\left(X_{h}, \sigma_{h}\right)$ is topologically conjugate to $(X, \sigma)$. 
Proposition 3.2. There exists $g: S^{3} \rightarrow S^{3}$, a $C^{1}$-diffeomorphism of the 3-sphere which has an attractor $\Lambda$ such that $(\Lambda, g)$ is topologically conjugate to $\left(X_{h}, \sigma_{h}\right)$.

(I) Proof of Proposition 3.1. We have to show that there exists a homeomorphism $\varphi_{0}$ from $X$ onto $X_{h}$ such that $\varphi_{0} \circ \sigma=\sigma_{h} \circ \varphi_{0}$. To do this we need several Lemmas.

Let $T^{1}=\boldsymbol{R} / \boldsymbol{Z}$ and denote the natural projection by $\pi_{0}: R \rightarrow T^{1}$. For each $x \in T^{1}$ there is a unique $t_{x} \in[0,1]$ with $\pi_{0}\left(t_{x}\right)=x$. Hence the continuous map $p_{0}: T^{1} \rightarrow I$ is well defined by $p_{0}(x)=1-\left|2 t_{x}-1\right|$. Consider the continuous map $\bar{\eta}(x)=2 x-(2 \pi)^{-1} \sin (2 \pi x)(x \in R)$ and denote by $\eta: T^{1} \rightarrow T^{1}$ the factor of $\bar{\eta}$ under $\pi_{0}$. Let $\zeta$ denote the endomorphism of $T^{1}$ defined by $\zeta(x)=2 x\left(x \in T^{1}\right)$.

(L.2) (i) $p_{0}$ is an open map. (ii) $p_{0}(x)=p_{0}(-x)\left(x \in T^{1}\right)$. (iii) $p_{0} \circ \zeta=$ $f \circ p_{0}$. (iv) $p_{0} \circ \eta=h \circ p_{0}$. (v) $\bar{\eta}(x)+\bar{\eta}(1-x)=2 \quad(x \in R)$. (vi) For every nonempty open set $U$ in $T^{1}$, there exists an integer $N>0$ such that $\eta^{N}(U)=T^{1}$.

Proof. (i) $\sim(v)$ are easy. (vi) follows from the fact that $\bar{\eta}^{\prime}(x)>1$ for every $x \in \boldsymbol{R}-\boldsymbol{Z}$.

We denote by $C^{0}(Y)$ the set of all continuous maps from a topological space $Y$ to itself. For each $\alpha \in C^{0}\left(T^{1}\right)$, we denote by $\bar{\alpha} \in C^{0}(R)$ a lift of $\alpha$. Then it is well known (P. 64 of [9]) that, for every $x \in \boldsymbol{R}$ and $n \in \boldsymbol{Z}$ with $n \neq 0$, the number $(1 / n)(\bar{\alpha}(x+n)-\bar{\alpha}(x))$ is an integer, and that this integer is independent of the choice of $x$ and $n$. Such an integer is called the degree of $\alpha$ and denoted by $\operatorname{deg}(\alpha)$. A map $\alpha \in C^{0}\left(T^{1}\right)$ is said to be monotone if a lift $\bar{\alpha}$ satisfies $\bar{\alpha}\left(x_{1}\right) \geqq \bar{\alpha}\left(x_{2}\right)$ for every $x_{1}, x_{2} \in \boldsymbol{R}$ with $x_{1} \geqq x_{2}$ (this definition is obviously independent of the choice of $\bar{\alpha}$ ).

(L.3) (i) $\operatorname{deg}(\zeta)=2$.

(ii) $\operatorname{deg}(\eta)=2$.

(iii) $\eta$ is monotone.

Proof. Obvious.

(L.4) There exists a homeomorphism $\alpha \in C^{0}\left(T^{1}\right)$ satisfying and

(i ) $\alpha(x)+\alpha(-x)=0\left(x \in T^{1}\right)$

(ii) $\alpha \circ \eta=\zeta \circ \alpha$.

Proof. Define

$H=\left\{\alpha \in C^{0}\left(T^{1}\right): \alpha\right.$ is monotone and satisfies $\left.\operatorname{deg}(\alpha)=1\right\}$ 
and

$V=\left\{\bar{\alpha} \in C^{0}(R): \bar{\alpha}\right.$ is a lift of some $\left.\alpha \in H . \quad \bar{\alpha}(x)+\bar{\alpha}(1-x)=1(x \in R)\right\}$. Since $\alpha \in H$ is degree-one, the metric function $D$ of $V$ is defined by

$$
D(\bar{\alpha}, \bar{\beta})=\max \{d(\bar{\alpha}(x), \bar{\beta}(x)): x \in[0,1]\} \quad \text { for } \quad \bar{\alpha}, \bar{\beta} \in V,
$$

where $d$ denotes the euclidian metric of $\boldsymbol{R}$.

We claim that $V$ is a complete metric space. Indeed, if $\left\{\bar{\alpha}_{i}\right\}$ is a Cauchy sequence with respect to $D$, then $\left\{\bar{\alpha}_{i}\right\}$ uniformly converges to some $\bar{\alpha} \in C^{0}(R)$. Since a uniform limit of lifts of degree-one maps is itself a lift, $\bar{\alpha}$ is a lift of some $\alpha_{0} \in C^{0}\left(T^{1}\right)$. As $\left\{\bar{\alpha}_{i}\right\} \subset V, \alpha_{0}$ is monotone and degree-one. Also $\bar{\alpha}$ satisfies $\bar{\alpha}(x)+\bar{\alpha}(1-x)=1 \quad(x \in R)$. Hence $\bar{\alpha}$ belongs to $V$, i.e. $V$ is complete.

Let $\bar{\zeta}$ be the lift of $\zeta$ defined by $\bar{\zeta}(x)=2 x(x \in R)$. Define the map $T: V \rightarrow C^{0}(R)$ by $T(\bar{\alpha})=\bar{\zeta}^{-1} \circ \bar{\alpha} \circ \bar{\eta}$. We claim that $T$ is a contraction map on $V$. Let $\bar{\alpha} \in V$ be given. Since $\operatorname{deg}(\eta)=2$ and $\operatorname{deg}(\alpha)=1$, we have $T(\bar{\alpha})(n+x)-T(\bar{\alpha})(x)=(1 / 2)(\bar{\alpha}(2 n+\bar{\eta}(x))-\bar{\alpha} \bar{\eta}(x))=n$ for every $x \in R$ and $n \in Z$. So $T(\bar{\alpha})$ is a lift of some $\alpha^{\prime} \in H$. Using $(\mathrm{L} .2(\mathrm{v}))$ and the equation $\bar{\alpha}(x)+\bar{\alpha}(1-x)=1$, we have

$$
\begin{gathered}
T(\bar{\alpha})(x)+T(\bar{\alpha})(1-x)=(1 / 2) \bar{\alpha} \bar{\eta}(x)+(1 / 2) \bar{\alpha}(2-\bar{\eta}(x)) \\
=(1 / 2)(\bar{\alpha} \bar{\eta}(x)+\bar{\alpha}(1-\bar{\eta}(x))+1)=1,
\end{gathered}
$$

so that $T(\bar{\alpha}) \in V$. This means $T(V) \subset V$. For every $\bar{\alpha}, \bar{\beta} \in V$, we have

$$
\begin{aligned}
D(T(\bar{\alpha}), T(\bar{\beta})) & =\max \left\{d\left(\bar{\zeta}^{-1} \bar{\alpha} \bar{\eta}(x), \bar{\zeta}^{-1} \bar{\beta} \bar{\eta}(x)\right): x \in[0,1]\right\} \\
& =(1 / 2) \max \{d(\bar{\alpha}(y), \bar{\beta}(y)): y=\bar{\eta}(x) \in[0,2]\} \\
& =(1 / 2) D(\bar{\alpha}, \bar{\beta}) .
\end{aligned}
$$

Therefore $T$ is a contraction map on $V$.

Since $V$ is complete, $T$ has a unique fixed point $\bar{\alpha}$ in $V$; i.e. $\bar{\alpha} \circ \bar{\eta}=$ $\bar{\zeta} \circ \bar{\alpha}$. Denote by $\alpha$ the factor of $\bar{\alpha}$ under $\pi_{0}$. It is easy to see that $\alpha \circ \eta=\zeta \circ \alpha$ and $\alpha(x)+\alpha(-x)=0\left(x \in T^{1}\right)$. To complete the proof of (L.4), it only remains to show that $\alpha$ is one-to-one. Assume that $x \neq y$ and $\alpha(x)=\alpha(y)$ for some $x, y \in T^{1}$. Then there is a nonempty open interval $U \subset T^{1}$ with $\alpha(U)=\alpha(x)$, because $\alpha$ is monotone and degree-one. By $(\mathrm{L} .2(\mathrm{vi}))$ one has $\eta^{N}(U)=T^{1}$ for some $N>0$. Hence $T^{1}=\alpha \eta^{N}(U)=\zeta^{N} \alpha(U)=$ $\zeta^{N} \alpha(x)$, which is a contradiction.

(L.5) There is a homeomorphism $\beta: I \rightarrow I$ such that $\beta \circ h=f \circ \beta$.

Proof. Let $\alpha$ and $p_{0}$ be as in (L.4) and (L.2) respectively. Suppose 
$p_{0}(x)=p_{0}(y)$ and $x \neq y$. Then one has $x=-y$, so that $p_{0} \alpha(x)=p_{0} \alpha(-y)=$ $p_{0}(-\alpha(y))=p_{0} \alpha(y)$ by (L.4(i)) and (L.2(ii)). Hence there is a map $\beta: I \rightarrow I$ such that $\beta \circ p_{0}=p_{0} \circ \alpha$. By (L.2(i)), $\beta$ is continuous. Similarly, since $\alpha$ is a homeomorphism, there is a continuous map $\beta^{\prime}: I \rightarrow I$ such that $\beta^{\prime} \circ p_{0}=p_{0} \circ \alpha^{-1}$. Then one has $\beta \circ \beta^{\prime} \circ p_{0}=\beta \circ p_{0} \circ \alpha^{-1}=p_{0} \circ \alpha \circ \alpha^{-1}=p_{0}$, and also $\beta^{\prime} \circ \beta \circ p_{0}=p_{0}$. Since $p_{0}$ is surjective, we have $\beta \circ \beta^{\prime}=\beta^{\prime} \circ \beta=$ id; i.e. $\beta$ is a homeomorphism. By (L.2(iv)), (L.4(ii)) and (L.2(iii)), it follows that $\beta \circ h \circ p_{0}=f \circ \beta \circ p_{0}$. Using $p_{0}\left(T^{1}\right)=I$, we get $\beta \circ h=f \circ \beta$.

Now we complete the proof of Proposition 3.1. Let $\beta$ be as in (L.5). Define the continuous map $\varphi_{0}: X \rightarrow I^{N}$ by $\varphi_{0}\left(\left(x_{i}\right)_{i \geq 1}\right)=\left(\beta^{-1} x_{i}\right)_{i \geq 1}$ for $\left(x_{i}\right)_{i \geq 1} \in X$. Since $h\left(\beta^{-1} x_{i+1}\right)=\beta^{-1} f\left(x_{i+1}\right)=\beta^{-1}\left(x_{i}\right)$ for every $\left(x_{i}\right)_{i \geq 1} \in X$, one has $\varphi_{0}(X) \subset X_{h}$. Since $\beta^{-1}: I \rightarrow I$ is a homeomorphism, $\varphi_{0}$ is a homeomorphism from $X$ onto $X_{h}$. Using the equation $\beta^{-1} \circ f=h \circ \beta^{-1}$, we have

$$
\varphi_{0} \sigma\left(\left(x_{i}\right)_{i \geq 1}\right)=\left(\beta^{-1} f\left(x_{i}\right)\right)_{i \geq 1}=\left(h \beta^{-1}\left(x_{i}\right)\right)_{i \geq 1}=\sigma_{h} \varphi_{0}\left(\left(x_{i}\right)_{i \geq 1}\right)
$$

for every $\left(x_{i}\right)_{i \geq 1} \in X$. Therefore $\left(X_{h}, \sigma_{h}\right)$ is topologically conjugate to $(X, \sigma)$. The proof is completed.

(II) Proof of Proposition 3.2. First of all we prepare some notation. Let $\kappa=\sinh ^{-1}(2)(\approx 1.44)$. Define

$$
M=\left\{(x, y, z) \in R^{3}:|x| \leqq \kappa, y \in[0, \pi],|z| \leqq \kappa\right\}
$$

and

$$
U_{0}=\bigcup_{v \in M}\left\{u \in R^{3}: d(u, v)<1 / 2\right\},
$$

where $d$ denotes the enclidian metric of $\boldsymbol{R}^{3}$. Then there exists a $C^{\infty}-\operatorname{map} \Phi: U_{0} \rightarrow \boldsymbol{R}^{3}$ such that

$$
\Phi(x, y, z)=\left\{\begin{array}{r}
(\sinh (x),-\cos (y) \cosh (z), \sin (y) \sinh (z)) \\
\text { for }(x, y, z) \in U_{0} \text { with } y \leqq \pi / 4, \\
(\sin (y) \sinh (x),-\cos (y) \cosh (x), \sinh (z)) \\
\text { for }(x, y, z) \in U_{0} \text { with } y \geqq 3 \pi / 4,
\end{array}\right.
$$

and $\Phi \mid M^{\prime}: M^{\prime} \rightarrow \Phi\left(M^{\prime}\right)$ is a $C^{\infty}$-diffeomorphism, where

$$
M^{\prime}=\{(x, y, z) \in M: \pi / 4 \leqq y \leqq 3 \pi / 4\} .
$$

Indeed, as such a $C^{\infty}$-map we can choose

$$
\begin{aligned}
\Phi(x, y, z)= & \chi_{0}(y)(\sinh (x),-\cos (y) \cosh (z), \sin (y) \sinh (z)) \\
& +\bar{\chi}_{0}(y)(\sin (y) \sinh (x),-\cos (y) \cosh (x), \sinh (z))
\end{aligned}
$$


where $\chi_{0}: R \rightarrow R$ is a monotone decreasing $C^{\infty}$-function such that $\chi_{0}(y)=1$ $(y \leqq \pi / 4)$ and $\chi_{0}(y)=0(y \geqq 3 \pi / 4)$, and $\bar{\chi}_{0}$ is defined by $\bar{\chi}_{0}(y)=1-\chi_{0}(y)(y \in R)$.

As an easy corollary the following holds.

(L.6) (i) Let $M(t) \quad(t \in[0, \pi])$ be the leaf of foliation defined by $M(t)=\{(x, y, z) \in M: y=t\}$, then $\Phi$ is one-to-one on $M-(M(0) \cup M(\pi))$.

(ii) $\Phi$ is a $C^{\infty}$-local diffeomorphism on $M-(\{(x, 0,0) \in M(0)\} \cup$ $\{(0, \pi, z) \in M(\pi)\})$.

(iii) There is a number $c>0$ such that $d(\Phi(u), \Phi(v)) \leqq c d(u, v)$ for every $u, v \in M$.

(iv) $\Phi(x, 0, z)=\Phi(x, 0,-z)$ for $(x, 0, z) \in M(0)$, and

$$
\Phi(x, \pi, z)=\Phi(-x, \pi, z) \text { for }(x, \pi, z) \in M(\pi) \text {. }
$$

(v) $\Phi$ is an open map.

(vi) Put $W=\Phi(M)$ (this is illustrated in Figure 1), then $\Phi\left(M_{0}\right)=$ $\operatorname{int}(W)$ where $M_{0}=\{(x, y, z) \in M:|x|<\kappa,|z|<\kappa\}$.

(vii) Put $W(t)=\Phi(M(t))(t \in[0, \pi])$. For each $u \in W$ there is a unique $t_{w} \in[0, \pi]$ with $u \in W\left(t_{u}\right)$. Then the map $p: W \rightarrow I$ defined by $p(u)=t_{w} / \pi$ is continuous.

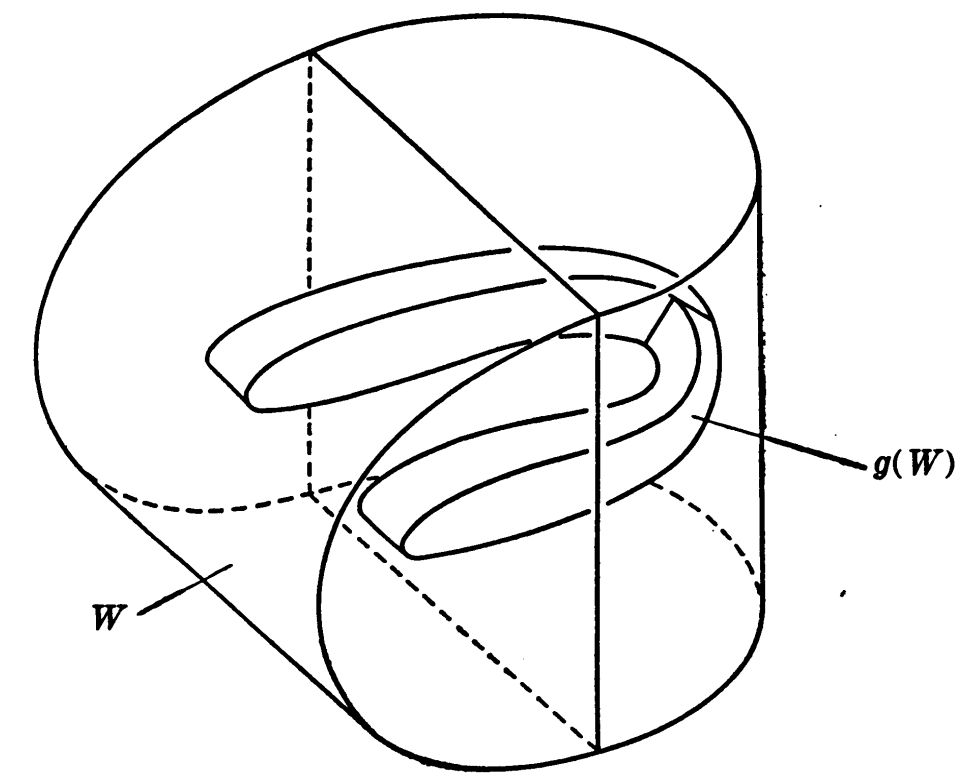

Figure 1

Proposition 3.3. Let $W$ and $\{W(t): t \in[0, \pi]\}$ be as above. Then there exists a continuous map $g: W \rightarrow W$ which satisfies the following conditions;

(1) let $h$ be a map as in (L.1) and define $h_{1}:(-\pi / 2,3 \pi / 2) \rightarrow R$ by $h_{1}(t)=\pi h(t / \pi)$, then $g(W(t)) \subset W\left(h_{1}(t)\right)$ for every $t \in[0, \pi]$, 
(2) $g(W) \subset \operatorname{int}(W)$,

(3) $\max _{t \in[0, \pi]} \operatorname{diam} g^{n}(W(t)) \rightarrow 0$ as $n \rightarrow \infty$,

(4) $g$ is one-to-one,

(5) $g$ is a $C^{\infty}$-local diffeomorphism on $W-\left(L_{1} \cup L_{2}\right)$ where

$$
L_{1}=\Phi(\{(x, 0,0) \in M(0)\}) \quad \text { and } \quad L_{2}=\Phi(\{(0, \pi, z) \in M(\pi)\}) \text {, }
$$

(6) $g$ is a $C^{1}$-local diffeomorphism on $L_{1} \cup L_{2}$,

(7) $g$ is isotopic to the identity map of $W$.

If Proposition 3.3 holds, then Proposition 3.2 is proved as follows. Let $g$ be the continuous map as in Proposition 3.3. From (4), (5) and (6) it follows that $g: W \rightarrow W$ is a $C^{1}$-diffeomorphism. We consider $W$ to be $W \subset R^{3} \subset S^{3}$. By the isotopy extension theorem (P. 180 of [7]), $g$ is extended to a $C^{1}$-diffeomorphism from $S^{3}$ onto itself. Denote the extended diffeomorphism by the same symbol $g$. Then $A=\bigcap_{n \geq 0} g^{n}(W)$ is a $g$ invariant compact set.

To show that $(\Lambda, g)$ is topologically conjugate to $\left(X_{h}, \sigma_{h}\right)$, let $p: W \rightarrow I$ be the continuous map as in (L.6(vii)). Then one has $h \circ p=p \circ g$ by (1). Since $h p g^{-(i+1)}(u)=p g^{-i}(u)$ for every $u \in \Lambda$ and $i \geqq 0$, the continuous map $\varphi: \Lambda \rightarrow X_{h}$ is well defined by $\varphi(u)=\left(p(u), p g^{-1}(u), p g^{-2}(u), \cdots\right)$. We claim that $\phi$ is one-to-one and onto; i.e. a homeomorphism. Indeed, if $p g^{-i}(u)=$ $p g^{-i}\left(u^{\prime}\right)$ for every $i \geqq 0$, then there are $t_{i} \in[0, \pi](i \geqq 0)$ such that $u, u^{\prime} \in$ $g^{i}\left(W\left(t_{i}\right)\right)$. By (3) one has $u=u^{\prime}$; i.e. $\varphi$ is one-to-one. To see $\varphi(\Lambda)=X_{h}$, let $\left(y_{i}\right)_{t \geq 1} \in X_{h}$ be given. It is easy to see that $\pi y_{i}=h_{1}\left(\pi y_{i+1}\right)$ for each $i \geqq 1$. Hence one has $g^{i}\left(W\left(\pi y_{i+1}\right)\right) \subset g^{i-1}\left(W\left(\pi y_{i}\right)\right)(i \geqq 1)$ by (1). By (3) there is $u_{y} \in \Lambda$ with $\left\{u_{y}\right\}=\bigcap_{i \geq 1} g^{i}\left(W\left(\pi y_{i+1}\right)\right)$. Since $\varphi\left(u_{y}\right)=\left(p g^{-i+1}\left(u_{y}\right)\right)_{i \geq 1}=\left(y_{i}\right)_{i \geq 1} \in$ $\varphi(\Lambda), \phi$ is onto. Since $\sigma \varphi(u)=\left(h p g^{-i+1}(u)\right)_{i \geq 1}=\left(p g^{-i+2}(u)\right)_{i \geq 1}=\varphi g(u)$ for every $u \in \Lambda,(\Lambda, g)$ is topologically conjugate to $\left(X_{h}, \sigma_{h}\right)$ under $\varphi$.

$(\Lambda, g)$ satisfies specification since so does $\left(X_{h}, \sigma_{h}\right)$ (by combining Theorem 1(B) and Proposition 3.1). Obviously $(\Lambda, g)$ is topologically transitive. Hence $\Lambda$ is an attractor of $g$ by (2). This prove Proposition 3.2

It remains only to prove Proposition 3.3.

(III) Proof of Proposition 3.3. We must construct a continuous map $g$ satisfying the conditions $(1) \sim(7)$. To do this we define several functions.

(L.7) Let $h_{2}: \boldsymbol{R} \rightarrow \boldsymbol{R}$ be a $C^{\infty}$-function such that

(i) $h_{2}(-t)=-h_{2}(t)(t \in R), \quad$ (ii) $\quad h_{2}(\kappa)=\kappa / 3$,

(iii) $h_{2}^{\prime}(0)=1$ and $0<h_{2}^{\prime}(t)<1 \quad(t \neq 0)$, 
(iv) $h_{2}^{\prime \prime}(t)<0(t>0)$,

(v) $\sqrt{\left(h_{2}(t)\right)^{2}+\left(h_{2}(s)\right)^{2}} \leqq \sqrt{2} h_{2}\left(\sqrt{t^{2}+s^{2}} / \sqrt{2}\right) \quad\left((t, s) \in R^{2}\right)$.

(As such a function, we can choose $h_{2}(t)=\lambda \tan ^{-1}(t / \lambda)$ where $\lambda$ is the root of $\tan (\kappa /(3 \lambda))=\kappa / \lambda$ with $0<\lambda<\pi / 2 ; \lambda \approx 0.306$.) Then one obtains

(vi) $h_{2}(t)=t+o\left(t^{2}\right), \quad$ (vii) $h_{2}\left(t_{1}\right)<h_{2}\left(t_{2}\right)\left(t_{1}<t_{2}\right)$,

(viii) $\quad \lim _{n \rightarrow \infty} h_{2}^{n}(t)=0 \quad(t \in \boldsymbol{R}), \quad(\mathrm{ix}) \quad\left|h_{2}(t)\right| \geqq|t| / 3 \quad(|t| \leqq \kappa)$,

(x) $\left|h_{2}(t)-h_{2}\left(t^{\prime}\right)\right| \leqq 2 h_{2}\left(\left|t-t^{\prime}\right| / 2\right)\left(t, t^{\prime} \in R\right)$.

Let $h_{0}$ define by $h_{0}(y)=2 y-(1 / 2) \sin (2 y) \quad(y \in R)$. Recall the map $h_{1}$ as in (1). We remark that $h_{1}(y)=h_{0}(y)$ on $(-\pi / 2, \pi / 2]$ and $h_{1}(y)=2 \pi-h_{0}(y)$ on $[\pi / 2,3 \pi / 2)$. Choose a constant $\alpha>0$ such that $h_{0}((\pi / 2)-\alpha)>3 \pi / 4$. Put $M_{1}=U_{t \in[0, \pi / 2]} M(t)$ and $M_{2}=\cup_{t \in[\pi / 2, \pi]} M(t)$. We denote by $U_{i}$ the $\alpha$-open neighborhood of $M_{i}$ in $R^{3}(i=1,2)$. Take a monotone decreasing $C^{\infty}$ function $\chi_{1}: R \rightarrow R$ such that

$$
\chi_{1}(y)=1 \quad(y \leqq \pi / 4) \quad \text { and } \quad \chi_{1}(y)=0 \quad(y \geqq(\pi / 2)-\alpha),
$$

and a monotone increasing $C^{\infty}$-function $\chi_{2}: R \rightarrow R$ such that

$$
\chi_{2}(y)=0 \quad(y \leqq(\pi / 2)+\alpha) \quad \text { and } \quad \chi_{2}(y)=1 \quad(y \geqq 3 \pi / 4) .
$$

Put $\bar{\chi}_{i}(y)=1-\chi_{i}(y)(i=1,2)$. We define two $C^{\infty}$-diffeomorphisms $G_{i}: U_{i} \rightarrow R^{3}$ $(i=1,2)$ by

$$
\begin{aligned}
G_{1}(x, y, z)= & \chi_{1}(y)\left(\frac{x}{3}-\frac{\kappa}{2}, h_{0}(y), h_{2}(z)\right) \\
& +\bar{\chi}_{1}(y)\left(\frac{1}{3 \sqrt{2}}(x-z)-\frac{\kappa}{2}, h_{0}(y), \frac{1}{3 \sqrt{2}}(x+z)\right)
\end{aligned}
$$

and

$$
\begin{aligned}
G_{2}(x, y, z)= & \chi_{2}(y)\left(\frac{z}{3}+\frac{\kappa}{2}, 2 \pi-h_{0}(y), h_{2}(x)\right) \\
& +\bar{\chi}_{2}(y)\left(\frac{1}{3 \sqrt{2}}(z-x)+\frac{\kappa}{2}, 2 \pi-h_{0}(y), \frac{1}{3 \sqrt{2}}(z+x)\right) .
\end{aligned}
$$

By the definitions of $G_{i}$ and $M_{i}$ one has $G_{i}\left(M_{i}\right) \subset M$ for $i=1,2$. Take an open neighborhood $U_{i}^{\prime} \subset U_{i}$ of $M_{i}$ such that $G_{i}\left(U_{i}^{\prime}\right) \subset U_{0}(i=1,2)$. We define the map $G: U_{1}^{\prime} \cup U_{2}^{\prime} \rightarrow R^{3}$ by

$$
G=G_{1} \quad \text { on } \quad\left\{(x, y, z) \in U_{1}^{\prime}: y \leqq \pi / 2\right\}
$$

and

$$
G=G_{2} \quad \text { on } \quad\left\{(x, y, z) \in U_{2}^{\prime}: y>\pi / 2\right\}
$$


Notice that $G$ is not continuous at $(x, \pi / 2, z) \in U_{1}^{\prime} \cap U_{2}^{\prime}$. Nevertheless, the composition $\Phi \circ G: U_{1}^{\prime} \cup U_{2}^{\prime} \rightarrow R^{3}$ is a $C^{\infty}$-map. Because, for $(x, y, z) \epsilon$ $U_{1}^{\prime} \cap U_{2}^{\prime}$, taking account of the inequalities

$$
|y-(\pi / 2)|<\alpha, \quad 3 \pi / 4<h_{0}(y)<\pi+\frac{1}{2} \quad \text { and } \quad 3 \pi / 4<2 \pi-h_{0}(y)<\pi+\frac{1}{2},
$$

one can easily verify that the definitions of $G_{1}, G_{2}$ and $\Phi$ imply the relation

$$
\begin{array}{r}
\Phi G_{1}(x, y, z)=\Phi G_{2}(x, y, z)=\left(\sin \left(h_{0}(y)\right) \sinh \left(\frac{1}{3 \sqrt{2}}(x-z)-\frac{\kappa}{2}\right),\right. \\
\left.-\cos \left(h_{0}(y)\right) \cosh \left(\frac{1}{3 \sqrt{2}}(x-z)-\frac{\kappa}{2}\right), \sinh \left(\frac{1}{3 \sqrt{2}}(x+z)\right)\right) .
\end{array}
$$

(L.8) (i) $G(M(t)) \subset\left\{\left(x, h_{1}(t), z\right) \in M:|x|<\kappa,|z|<\kappa\right\}$ for $t \in[0, \pi]$.

(ii) $\Phi \circ G$ is one-to-one on $M-(M(0) \cup M(\pi))$.

(iii) $\Phi \circ G$ is a $C^{\infty}$-local diffeomorphism on

$$
M-(\{(x, 0,0) \in M(0)\} \cup\{(0, \pi, z) \in M(\pi)\}) .
$$

Proof. (i) follows from $h_{1}(t)=h_{0}(t) \quad(t \leqq \pi / 2)$ and $h_{1}(t)=2 \pi-h_{0}(t) \quad(t>$ $\pi / 2)$. (ii) and (iii) follow immediately from the definitions of $\Phi$ and $G$.

Now we show the existence of a map $g: W \rightarrow W$ with $g \circ \Phi=\Phi \circ G$. Suppose that $\Phi(x, y, z)=\Phi\left(x^{\prime}, y^{\prime}, z^{\prime}\right)$ and $(x, y, z) \neq\left(x^{\prime}, y^{\prime}, z^{\prime}\right)$. By (L.6(i)) we have either $x=x^{\prime}, y=y^{\prime}=0$ and $z=z^{\prime}$, or $x=-x^{\prime}, y=y^{\prime}=\pi$ and $z=z^{\prime}$. Hence, by (L.6(iv)) and (L.7(i)), we have

$$
\Phi G(x, 0, z)=\Phi\left(\frac{x}{3}-\frac{\kappa}{2}, 0, h_{2}(z)\right)=\Phi\left(\frac{x}{3}-\frac{\kappa}{2}, 0, h_{2}(-z)\right)=\Phi G(x, 0,-z) .
$$

Similarly $\Phi G(x, \pi, z)=\Phi G(-x, \pi, z)$ holds. Consequently we have $\Phi G(x, y, z)=\Phi G\left(x^{\prime}, y^{\prime}, z^{\prime}\right)$. This implies that there exists a map $g$ such that $g \circ \Phi=\Phi \circ G$. The image $g(W)$ is illustrated in Figure 1 .

In order to prove Proposition 3.3, it remains only to show that $g$ is continuous and satisfies the conditions (1) ( (7).

(L.9) (i) $g$ is continuous.

(ii) $g$ satisfies the conditions (1) (5).

Proof. (i) follows from (L.6(v)). $g$ satisfies (1) by (L.8(i)), (2) by (L.6(vi)) and (L.8(i)), and (5) by (L.8(iii)). We prove that $g$ satisfies (3). Let $y \in[0, \pi]$ be given. Suppose $y \leqq \pi / 2$. Then, for every $u=(x, y, z)$ 
and $u^{\prime}=\left(x^{\prime}, y, z^{\prime}\right)$ in $M(y)$, we have

$$
\begin{aligned}
d\left(G(u), G\left(u^{\prime}\right)\right) & \leqq \chi_{1}(y)\left\|\left(\frac{x-x^{\prime}}{3}, 0, h_{2}(z)-h_{2}\left(z^{\prime}\right)\right)\right\|+\bar{\chi}_{1}(y)\left\|\left(\frac{x-x^{\prime}}{3}, 0, \frac{z-z^{\prime}}{3}\right)\right\| \\
& \leqq 2\left\|\left(h_{2}\left(\frac{x-x^{\prime}}{3}\right), 0, h_{2}\left(\frac{z-z^{\prime}}{2}\right)\right)\right\| \quad(\text { by (ix) and (x) in (L.7)) } \\
& \leqq 2^{3 / 2} h_{2}\left(2^{-3 / 2} d\left(u, u^{\prime}\right)\right) \quad \text { (by (L.7(v))) } .
\end{aligned}
$$

Similarly, for $y>\pi / 2$, we have $d\left(G(u), G\left(u^{\prime}\right)\right) \leqq 2^{3 / 2} h_{2}\left(2^{-8 / 2} d\left(u, u^{\prime}\right)\right)$ for every $u, u^{\prime} \in M(y)$. Hence it follows that

$$
\begin{gathered}
2^{-3 / 2} \operatorname{diam} G^{n}(M(y)) \leqq h_{2}\left(2^{-8 / 2} \operatorname{diam} G^{n-1}(M(y))\right) \leqq \cdots \\
\leqq h_{2}^{n}\left(2^{-3 / 2} \operatorname{diam} M(y)\right)=h_{2}^{n}(x)
\end{gathered}
$$

for every $y \in[0, \pi]$ and $n>0$. From this we get

$$
\begin{array}{rlrl}
\max _{y \in[0, \pi]} \operatorname{diam} g^{n}(W(y))=\max _{y \in[0, \pi]} \operatorname{diam} \Phi \circ G^{n}(M(y)) \\
& \leqq c \cdot \max _{y \in[0, \pi]} \operatorname{diam} G^{n}(M(y)) & & (\text { by }(\text { L.6(iii))) } \\
& \left.\leqq 2^{3 / 2} c h_{2}^{n}(\kappa) \longrightarrow 0 \text { (as } n \longrightarrow \infty\right) & & (\text { by }(\text { L.7(viii))); }
\end{array}
$$

i.e. $g$ satisfies (3).

We prove that $g$ satisfies (4). By (L.6(i)) and (L.8(ii)), $g$ is one-toone on $W-(W(0) \cup W(\pi))$. Let $(r, s, 0),\left(r^{\prime}, s^{\prime}, 0\right) \in W(0)$ satisfy $g(r, s, 0)=$ $g\left(r^{\prime}, s^{\prime}, 0\right)$. There exist $(x, 0, z)$ and $\left(x^{\prime}, 0, z^{\prime}\right)$ in $M(0)$ such that $\Phi(x, 0, z)=$ $(r, s, 0)$ and $\Phi\left(x^{\prime}, 0, z^{\prime}\right)=\left(r^{\prime}, s^{\prime}, 0\right)$. Since $g \circ \Phi=\Phi \circ G$, we have

$$
\left(\sinh \left(\frac{x}{3}-\frac{\kappa}{2}\right),-\cosh \left(h_{2}(z)\right), 0\right)=\left(\sinh \left(\frac{x^{\prime}}{3}-\frac{\kappa}{2}\right),-\cosh \left(h_{2}\left(z^{\prime}\right)\right), 0\right) .
$$

By (L.7(i)) we get eigher $x=x^{\prime}$ and $z=z^{\prime}$, or $x=x^{\prime}$ and $z=-z^{\prime}$. In any case, $\Phi(x, 0, z)=\Phi\left(x^{\prime}, 0, z^{\prime}\right)$; i.e. $(r, s, 0)=\left(r^{\prime}, s^{\prime}, 0\right)$. Hence $g$ is one-to-one on $W(0)$. Similarly it follows that $g$ is one-to-one on $W(\pi)$. Since $g(W-(W(0) \cup W(\pi))) \cap g(W(0) \cup W(\pi))=\varnothing, g$ is one-to-one on $W$; i.e. $g$ satisfies (4).

\section{(L.10) $g$ satisfies (6) and (7).}

Proof. First we prove that $g$ is a $C^{1}$-local diffeomorphism on $L_{1}$. Let $v_{0}=\left(r_{0},-1,0\right)$ be a point in $L_{1}$ and $v=(r, s, t)$ a point sufficiently near $v_{0}$ with $v \neq v_{0}$. Take a point $u_{0}=\left(x_{0}, 0,0\right)$ such that $\Phi\left(u_{0}\right)=v_{0}$. There is a point $u=(x, y, z)$ in $U_{0}$ such that $\Phi(u)=v$. Since $u$ is also sufficiently near $u_{0}$ by $(\mathrm{L} .6(\mathrm{v}))$, we may asume that $-\pi / 4 \leqq y \leqq \pi / 4$. Then we have 


$$
(r, s, t)=(\sinh (x),-\cos (y) \cosh (z), \sin (y) \sinh (z))
$$

and

$$
\begin{aligned}
g(v) & =\left(g_{1}, g_{2}, g_{8}\right) \\
& =\left(\sin \left(\frac{x}{3}-\frac{\kappa}{2}\right),-\cos \left(h_{1}(y)\right) \cosh \left(h_{2}(z)\right), \sin \left(h_{1}(y)\right) \sinh \left(h_{2}(z)\right)\right) .
\end{aligned}
$$

Hence

$$
\frac{d g_{1}}{d r}=\frac{d g_{1}}{d x} / \frac{d r}{d x} \longrightarrow a_{0} \quad\left(\text { as } x \longrightarrow x_{0} \text {; i.e. } r \longrightarrow r_{0}\right)
$$

where $a_{0}=\left\{\cosh \left(\left(x_{0} / 3\right)-(\kappa / 2)\right)\right\} /\left\{3 \cosh \left(x_{0}\right)\right\}>0$. Using (L.1(iv)) and (L.7(vi)), we get

$$
\begin{aligned}
\lim _{(s, t) \rightarrow(-1,0)} & \frac{\left\|\left(g_{2}(v), g_{3}(v)\right)-\left(g_{2}\left(v_{0}\right), g_{8}\left(v_{0}\right)\right)-(s+1, t)\right\|}{\|(s+1, t)\|} \\
= & \lim _{(y, z) \rightarrow(0,0)}\left[\frac{\left\{o\left(z^{2}\right) \cos (y)+o\left(y^{2}\right) \cosh (z)+o\left(y^{2}\right) o\left(z^{2}\right)\right\}^{2}}{\{\cosh (z)-\cos (y)\}^{2}}\right. \\
& \left.+\frac{\left\{o\left(z^{2}\right) \sin (y)+o\left(y^{2}\right) \sinh (z)+o\left(y^{2}\right) o\left(z^{2}\right)\right\}^{2}}{\{\cosh (z)-\cos (y)\}^{2}}\right]^{1 / 2} \\
= & 0 .
\end{aligned}
$$

Therefore $g$ is differentiable at $\left(r_{0},-1,0\right)$ and one has

$$
D g\left(r_{0},-1,0\right)=\left[\begin{array}{lll}
a_{0} & & 0 \\
& 1 & \\
0 & & 1
\end{array}\right] \text { and } J g\left(r_{0},-1,0\right)=a_{0}>0 \text {. }
$$

From an easy calculation it follows that

$$
\frac{\partial\left(g_{1}, g_{2}, g_{3}\right)}{\partial(r, s, t)}=\frac{\partial\left(g_{1}, g_{2}, g_{3}\right)}{\partial(x, y, z)} \cdot\left[\frac{\partial(r, s, t)}{\partial(x, y, z)}\right]^{-1} \longrightarrow\left[\begin{array}{lll}
a_{0} & & 0 \\
& 1 & \\
0 & & 1
\end{array}\right] \quad\left(\text { as } u \longrightarrow u_{0}\right)
$$

This implies that $g$ is a $C^{1}$-local diffeomorphism on $L_{1}$. Similarly we can prove that $g$ is a $C^{1}$-local diffeomorphism on $L_{2}$. Therefore $g$ satisfies (6).

From (4), (5) and (6), $g$ is a $C^{1}$-diffeomorphism from $W$ into $R^{3}$. Since $W$ is a closed ball in $R^{3}$ and $J g(u)>0$ holds at $u=\left(r_{0},-1,0\right) \in W$, $g$ is orientation preserving. Therefore $g$ is isotopic to the identity map (P. 117 of [7]); i.e. $g$ satisfies (7). The proof is completed. 


\section{References}

[1] N. AOKI and M. Dateyama, The relationship between algebraic number and expansiveness of group automorphisms, to appear in Fund. Math..

[2] N. AOKI, M. Dateyama and M. KomURo, Solenoidal automorphisms with specifications, Monatsh. Math., 93 (1982), 79-110.

[3] R. Bow EN, Periodic points and measures for axiom A diffeomorphisms, Trans. Amer. Math. Soc., 154 (1971), 377-397.

[4] R. BowEN, Some systems with unique equilibrium state, Math. Systems Theory, 8 (1974), 193-202.

[5] M. Denker, C. Grillenderger and K. Sigmund, Ergodic Theory on Compact Spaces, Lecture Notes in Math., 527, Springer, 1976.

[6] M. HÉnON, A two-dimensional mapping with a strange attractor, Comm. Math. Phps., 50 (1976), 69-77.

[7] M. W. HIRSCH, Differential Topology, Graduate Texts in Math., 33, Springer, 1976.

[8] D. LiND, Ergodic group automorphisms and specification, Lecture Notes Math., 729 (edited by Denker and Jacobs) Ergodic Theory, Springer, 1979, 93-104.

[9] Z. Nitecki, Differentiable Dynamics, The MIT Press, Cambridge Ma., 1971.

[10] K. Sigmund, On dynamical systems with the specification property, Trans. Amer. Math. Soc., 190 (1974), 285-299.

[11] S. Smale, Differentiable dynamical systems, Bull. Amer. Math. Soc., 73 (1967), 747-817.

[12] R. F. Williams, One-dimensional non-wandering sets, Topology, 6 (1967), 473-487.

[13] R. F. Williams, Classification of one-dimensional attractors, Global Analysis, Proceedings of Symposia in Pure Math., 14 (1970), Amer. Math. Soc., Providence, 341-361.

Present Address:

Department of Mathematics

Faculty of Sciences

Tokyo METropolitan University

Fukazawa, Setagaya-ku, Tokyo 158 